\title{
Cronobacter, the emergent bacterial pathogen Enterobacter sakazakii comes of age; MLST and whole genome sequence analysis
}

\author{
Stephen J Forsythe ${ }^{1 *}$, Benjamin Dickins ${ }^{1}$ and Keith A Jolley ${ }^{2}$
}

\begin{abstract}
Background: Following the association of Cronobacter spp. to several publicized fatal outbreaks in neonatal intensive care units of meningitis and necrotising enterocolitis, the World Health Organization (WHO) in 2004 requested the establishment of a molecular typing scheme to enable the international control of the organism. This paper presents the application of Next Generation Sequencing (NGS) to Cronobacter which has led to the establishment of the Cronobacter PubMLST genome and sequence definition database (http://pubmlst.org/ cronobacter/) containing over 1000 isolates with metadata along with the recognition of specific clonal lineages linked to neonatal meningitis and adult infections

Results: Whole genome sequencing and multilocus sequence typing (MLST) has supports the formal recognition of the genus Cronobacter composed of seven species to replace the former single species Enterobacter sakazakii. Applying the 7-loci MLST scheme to 1007 strains revealed 298 definable sequence types, yet only C. sakazakii clonal complex 4 (CC4) was principally associated with neonatal meningitis. This clonal lineage has been confirmed using ribosomal-MLST (51-loci) and whole genome-MLST (1865 loci) to analyse 107 whole genomes via the Cronobacter PubMLST database. This database has enabled the retrospective analysis of historic cases and outbreaks following re-identification of those strains.

Conclusions: The Cronobacter PubMLST database offers a central, open access, reliable sequence-based repository for researchers. It has the capacity to create new analysis schemes 'on the fly', and to integrate metadata (source, geographic distribution, clinical presentation). It is also expandable and adaptable to changes in taxonomy, and able to support the development of reliable detection methods of use to industry and regulatory authorities. Therefore it meets the WHO (2004) request for the establishment of a typing scheme for this emergent bacterial pathogen. Whole genome sequencing has additionally shown a range of potential virulence and environmental fitness traits which may account for the association of C. sakazakii CC4 pathogenicity, and propensity for neonatal CNS.
\end{abstract}

Keywords: Emergent bacterial pathogen, Cronobacter, MLST, Genomic analysis

\section{Background}

Next generation sequencing and multi-allelic profiling of emergent bacterial pathogens

Sequence-based methods for bacterial identification started with single locus sequencing, such as the $16 \mathrm{~S}$ rRNA gene. This can differentiate isolates between phylum to genus level and often to species level, but no further [1]. The application of multilocus sequence typing (MLST), typically 7 loci, enables the recognition of bacterial sequence types

\footnotetext{
* Correspondence: Stephen.forsythe@ntu.ac.uk

'School of Science and Technology, Nottingham Trent University, Clifton Lane, Nottingham NG11 8NS, UK

Full list of author information is available at the end of the article
}

(STs) and clonal complexes (CCs). These loci were initially sequenced individually with specifically designed primers. Nowadays, the application of Next Generation Sequencing (NGS) has facilitated whole genome sequencing for the equivalent cost of 7-loci and therefore has greatly increased the number of loci that can be used for strain discrimination and definition. The use of ribosomal MLST (rMLST) with 53 loci defined has already been used to define established bacterial pathogens to the strain level [2]. The use of whole genome MLST ( $>500$ loci) to clone level is now possible and has been applied in epidemiological studies for pathogen typing, e.g. Neisseria meningitidis, 
methicillin-resistant Staphylococcus aureus and bacterial population genomics of Campylobacter [3-6].

\section{The emergent bacterial pathogen Cronobacter and the FAO-WHO call for molecular typing methods}

Bacteria causing neonatal infections naturally attract attention and invoke a sense of injustice. Following the recognition of E. sakazakii (former name of Cronobacter genus) as the causative agent of fatal infections of neonates due to contaminated reconstituted infant formula [7-9] there were three FAO-WHO risk assessment expert group meetings [10-12] on the microbiological safety of these products on the international market. This was the first occasion that the WHO had aimed to control a foodborne bacterial pathogen. However at that time there had been few studies of relevance to help with imposing international control measures. Also the organism had undergone a number of taxonomic evaluations based on phenotypic tests resulting in confusing and conflicting information hampering the international control of this emergent bacterial pathogen by the development of specifc detection methods. Isolates had been variously described as Praschechia flavescens and 'yellow-pigmented Enterobacter cloacae, before a key study renaming them as $E$. sakazakii in 1980 and dividing the species into 15 biotypes [13]. Subsequently in order to reduce the risk of further neonatal infections, the FAO-WHO executive encouraged the setting up of appropriate detection and molecular typing schemes [10]. These are required for the adequate monitoring of sources and vehicles of the bacterium, in order to reduce the risk of neonatal exposure. These key points from the FAO-WHO executive summary are reproduced in Additional file 1: Box 1.

To date NGS of bacteria has primarily focused on well described organisms of clinical relevance such as Escherichia coli and Salmonella. This article, instead, considers the application of NGS to the newly emergent bacterial pathogen Cronobacter which was totally unlike Salmonella or E. coli with the lack of former information on its habitats, diversity, physiological or virulence traits The three tasks set by the FAO-WHO expert group (Additional file 1: Box 1) form the basis for this article to illustrate how using NGS has led to the establishment of the Cronobacter PubMLST genome database, and enabled the improved understanding and control for this emergent bacterial pathogen of international importance. Each task will be covered below as a discourse.

\section{Results and discussion}

\section{Multilocus sequence typing of Cronobacter spp. and the Cronobacter PubMLST database}

The need for an internationally validated detection and molecular typing method for the genus Cronobacter is needed given the severe outcomes of infections in neonates and the attributed link to contaminated powdered infant formula on the international market [10-12]. The Cronobacter PubMLST database includes the MLST profiling of isolates from all reported outbreaks around the world. Since the database also contains the metadata for over 1000 isolates an informed understanding of the diversity and sources of the organism can now be obtained. The MLST scheme covers all recognised species of Cronobacter genus, to better quantitate the intraspecific and interspecific diversity of the genus as well as potentially characterize the strains according to virulence groupings and source. It also enables the retrospective analysis of historic cases and outbreaks following re-identification of those strains which otherwise would have been lost due to taxonomic re-evaluations.

The organism initially was only known as a single species, Enterobacter sakazakii, composed of various biotypes based on phenotyping [13]. The application of $16 \mathrm{~S}$ rRNA and dnaG gene sequencing led to the initial recognition that the organism was in fact composed of several species, and formed a unique genus that was distinguishable from Enterobacter [14]. The new genus was named Cronobacter to reflect the association of the bacterium with infant death (Kronos - Greek mythological god) [15].

However 16S rRNA gene sequencing was unable to distinguish between all Cronobacter species and biotyping based on phenotypic tests gave subjective results. In order to overcome various limitations of phenotyping and $16 \mathrm{~S}$ rDNA sequence analysis, the Cronobacter seven-loci MLST scheme was developed [16,17]. The Cronobacter MLST scheme requires the partial sequence analysis of seven housekeeping genes: ATP synthase b chain $(a t p D)$, elongation factor $\mathrm{G}$ (fusA), glutaminyl tRNA synthetase $(g \ln S)$, glutamate synthase large subunit $(g l t B)$, DNA gyrase subunit B $(g y r B)$, translation initiation factor IF-2 $($ inf $B)$ and phosphoenolpyruvate synthase A (ppsA). When concatenated together these sequences provide 3036 nucleotides; the analysis of which is termed multilocus sequence analysis (MLSA). A MLSA phylogenetic tree of the Cronobacter genus and the closely related genera Franconibacter and Siccibacter is shown in Figure 1. A current description of the Cronobacter genus is given in Additional file 1: Box 2.

Before 2004 the number of Enterobacteriaceae (except for Salmonella) permitted in powdered infant formula was $<100 \mathrm{cfu} / \mathrm{g}$. These specifications had not been revised since 1979. However following the concern of the serious implications following Cronobacter infections of neonates, the microbiological criteria changed such that any member of the Cronobacter genus should not be detectable in $10 \mathrm{~g}$ test volume quantities of powdered infant formula [18]. Reliable detection of Cronobacter spp. has also been problematic due to the genus being poorly 


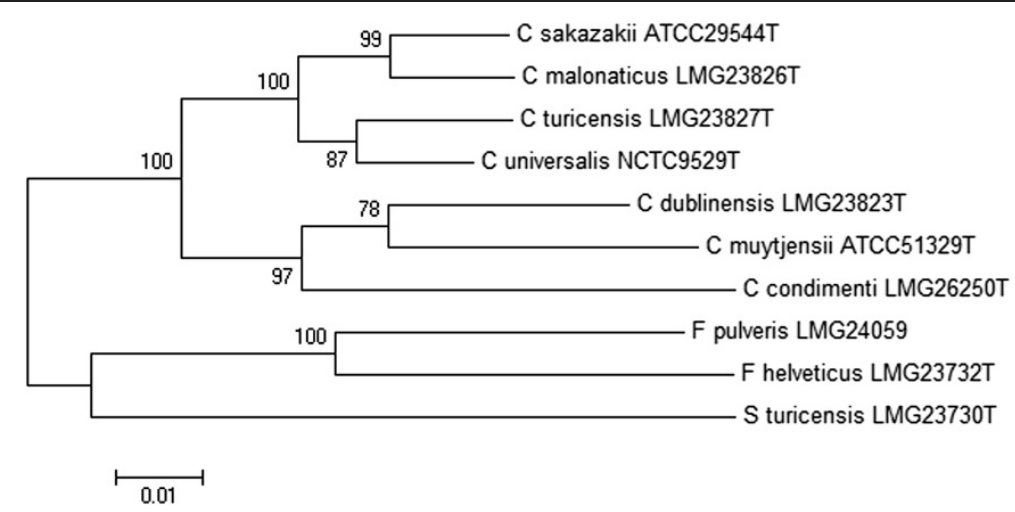

Figure 1 Maximum likelihood tree of the seven multilocus sequence typing loci (3036 base pair concatenated length) for the members of Cronobacter genus and closely related Franconibacter and Siccibacter genera. The tree was drawn using MEGA 6.05 (http://www. megasoftware.net/) with 1000 bootstrap replicates.

described. Prior to 2004, isolates were recovered using the general Enterobacteriaceae differential agar VRBGA, and then identified using a non-specific commercial phenotyping test kit resulting in considerable opportunity for false negative results [19]. While much of this topic is outside the scope of this article, it is pertinent to note that one biochemical trait that has predominated in the development of specific chromogenic agar is the constitutive expression of a maltose uptake mechanism and $\alpha$ -glucosidase [20]. This will be reconsidered in the last section with respect to taxonomy of the genus.

\section{Sources and vehicles of Cronobacter spp.}

Investigating 1007 isolate entries in the Cronobacter MLST database reveals for the first time the temporal, geographic and source diversity of the organism; Table 1. The earliest isolate (C. sakazakii NCIMB 8282) was from dried milk powder in 1950; the genome of which has now been published and is one of 107 genomes which can now be analysed via the PubMLST database [21].
Cronobacter strains have been isolated from 36 countries, and are from clinical (20.4\%), infant formula (21.6\%), food and food ingredients (14.2\%), environmental (35.15\%) and other sources such as water (4\%); Tables 1 and 2 . Cronobacter spp. isolation from plant material (wheat, rice, herbs, and spices) and various food products has previously been reported [22]. However, due to the history of concern, it is not surprising that a large portion of strains in the database are clinical or from powdered infant formula in origin.

The index of association $\left(\mathrm{I}_{\mathrm{A}}\right)$ is a measure of the linkage of a population. The $\mathrm{I}_{\mathrm{A}}$ values for the genus Cronobacter was found to be significantly greater than zero ( $p<0.001)$, indicating the presence of linkage disequilibrium or clonality $[16,17]$. Clonal complexes are the STs which share 3 or more loci to the central ST. This recognises there are variants of each allele, for example single and double locus variant (SLV, DLV). A number of these large clonal complexes are especially significant with respect to strain clustering according to their

Table 1 Summary of Cronobacter isolates in the Cronobacter PubMLST database

\begin{tabular}{|c|c|c|c|c|c|c|c|c|c|c|}
\hline \multirow[t]{2}{*}{ Species } & \multirow{2}{*}{$\begin{array}{l}\text { Number of } \\
\text { strains (\%) }\end{array}$} & \multirow{2}{*}{$\begin{array}{l}\text { Number } \\
\text { of STs }{ }^{a}\end{array}$} & \multirow{2}{*}{$\begin{array}{c}\text { Number } \\
\text { of genomes }\end{array}$} & \multirow{2}{*}{$\begin{array}{l}\text { Earliest } \\
\text { isolate }\end{array}$} & \multirow[t]{2}{*}{ Countries } & \multicolumn{5}{|c|}{ Source } \\
\hline & & & & & & Clinical & $\begin{array}{c}\text { Infant } \\
\text { formula }\end{array}$ & $\begin{array}{l}\text { Food and } \\
\text { ingredients }\end{array}$ & Environment & Other \\
\hline C. sakazakii & $726(72.1)$ & 155 & 73 & 1950 & 33 & $19.8^{b}$ & 23.8 & 32.8 & 16.3 & 7.3 \\
\hline C. malonaticus & $136(13.5)$ & 53 & 14 & 1973 & 17 & 36.0 & 16.2 & 36.8 & 7.4 & 3.7 \\
\hline C. dublinensis & $59(5.9)$ & 44 & 9 & 1956 & 11 & 5.1 & 13.6 & 47.5 & 6.8 & 27.1 \\
\hline C. turicensis & $41(4.1)$ & 26 & 6 & 1970 & 12 & 17.1 & 12.2 & 36.6 & 22.0 & 12.2 \\
\hline C. muytjensii & $35(3.5)$ & 14 & 2 & 1988 & 10 & 2.9 & 28.6 & 45.7 & 2.9 & 20.0 \\
\hline C. universalis & $9(0.9)$ & 5 & 2 & 1956 & 6 & 11.1 & 0.0 & 55.6 & 11.1 & 22.2 \\
\hline C. condimenti & $1(0.1)$ & 1 & 1 & 2010 & 1 & 0 & 0 & 100 & 0 & 0 \\
\hline Total & 1007 & 298 & 107 & & 36 & $20.4^{c}$ & 21.6 & 14.2 & 35.1 & 8.7 \\
\hline
\end{tabular}

a Sequence type.

${ }^{b}$ Percentage of species total.

'Percentage of genus total. 
Table 2 Profile of the source for the major sequence types in C. sakazakii and C. malonaticus

\begin{tabular}{|c|c|c|c|c|c|c|c|c|c|c|c|c|c|c|c|}
\hline \multirow{2}{*}{$\begin{array}{l}\text { Cronobacter } \\
\text { species }\end{array}$} & \multirow{2}{*}{$\begin{array}{l}\text { Clonal } \\
\text { group }\end{array}$} & \multirow{2}{*}{$\begin{array}{l}\text { Number of } \\
\text { profiled } \\
\text { isolates }\end{array}$} & \multirow{2}{*}{$\begin{array}{l}\text { Percentage } \\
\text { of species } \\
\text { isolates }\end{array}$} & \multicolumn{4}{|c|}{ Infant $(<1 \mathrm{y})^{\mathrm{b}}$} & \multirow{2}{*}{$\begin{array}{l}\text { Child } \\
(>1 \text { y) }\end{array}$} & \multirow{2}{*}{$\begin{array}{c}\text { Adult } \\
(>18 y)\end{array}$} & \multirow[t]{2}{*}{ Unknown $^{\mathrm{e}}$} & \multicolumn{5}{|c|}{ Source } \\
\hline & & & & Uncertain $^{c}$ & Meningitis & $\mathrm{NEC}^{\mathrm{d}}$ & Septicaemia & & & & $\begin{array}{c}\text { Clinical } \\
\text { total }\end{array}$ & $\begin{array}{c}\text { Infant } \\
\text { formula }\end{array}$ & $\begin{array}{c}\text { Food \& } \\
\text { ingredients }\end{array}$ & Environment & Others \\
\hline \multirow[t]{9}{*}{ C. sakazakii $(726)^{a}$} & CC4 & 195 & 26.9 & 4.6 & 10.0 & 5.1 & 4.1 & 2.1 & 16.9 & 2.0 & 45.1 & 24.6 & 8.2 & 16.9 & 5.6 \\
\hline & CC1 & 80 & 11.0 & 2.5 & 3.8 & 0.0 & 1.3 & 1.3 & 0.0 & 3.8 & 12.5 & 30.0 & 22.5 & 31.3 & 3.8 \\
\hline & CC8 & 35 & 4.8 & 5.7 & 0.0 & 0.0 & 0.0 & 5.7 & 0.0 & 20.0 & 31.4 & 17.1 & 37.1 & 5.7 & 8.6 \\
\hline & CC64 & 28 & 3.9 & 0.0 & 0.0 & 0.0 & 0.0 & 0.0 & 0.0 & 3.6 & 3.6 & 46.4 & 35.7 & 7.1 & 7.1 \\
\hline & CC45 & 25 & 3.4 & 0.0 & 0.0 & 0.0 & 0.0 & 0.0 & 0.0 & 4.0 & 4.0 & 8.0 & 52.0 & 32.0 & 4.0 \\
\hline & CC13 & 24 & 3.3 & 10.5 & 0.0 & 0.0 & 0.0 & 0.0 & 0.0 & 0.0 & 8.3 & 29.2 & 58.3 & 0.0 & 4.2 \\
\hline & CC3 & 19 & 2.6 & 13.3 & 0.0 & 0.0 & 0.0 & 0.0 & 0.0 & 0.0 & 10.5 & 31.6 & 36.8 & 15.8 & 5.3 \\
\hline & ST12 & 15 & 2.1 & 27.3 & 0.0 & 13.3 & 0.0 & 0.0 & 0.0 & 26.7 & 60.0 & 20.0 & 20.0 & 0.0 & 0.0 \\
\hline & CC83 & 11 & 1.5 & 0.0 & 0.0 & 0.0 & 9.1 & 0.0 & 0.0 & 0.0 & 9.1 & 18.2 & 9.1 & 63.6 & 0.0 \\
\hline C. malonaticus (136) & CC7 & 58 & 42.6 & 3.4 & 0.0 & 0.0 & 0.0 & 22.4 & 15.5 & 15.5 & 56.9 & 12.1 & 25.9 & 0.0 & 5.2 \\
\hline
\end{tabular}

${ }^{a}$ Number of strains for each species in PubMLST database. See Table 1.

${ }^{b}$ Percentage of strains in each clonal group.

"No clinical presentation for infant isolate known.

Necrotizing enterocolitis.

${ }^{\mathrm{e}} \mathrm{Clinical}$, unknown presentation and host information. 
isolation sources which will be considered in the next section.

The majority of Cronobacter infections, albeit less severe, are in the adult population [12]. An age profile of Cronobacter isolated using throat swabs from over 45,000 outpatients during the period 2005-2011 has been published [23]. The organism was isolated from every age group with a higher frequency from children less than 14 years of age. However the speciation and sequence type of these clinical isolates has not been published. As neonates are frequently fed reconstituted powdered infant formula (PIF), which is not a sterile product, this potential vector has been the focus of attention for reducing infection risk to neonates as the number of exposure routes is limited. It should be noted, however, that not all have been associated with reconstituted formula ingestion, and the organism has been isolated from the feeding tubes of neonates not receiving reconstituted powdered infant formula [24].

GoeBURST analysis shows the formation of clonal complexes among the 298 identified STs for the Cronobacter genus (Figure 2), and an uneven distribution of clinical isolates across both the species and sequence types $[17,25,26]$. Some of these large clonal complexes are especially significant with respect to strain clustering according to their isolation sources. All sequence types can be analysed from the open access database which is updated regularly, however for convenience only selected ones are considered in detail below.

Clonal complex 4 (CC4) comprises single and double loci variants of C. sakazakii ST4. Currently these strains comprise $19.4 \%(n=195)$ of the Cronobacter genus database. Forty-five percent (88/195) of these are clinical isolates and $24.6 \%(48 / 195)$ have been isolated from powdered infant formula; Table 2 . This analysis demonstrates the importance of this clonal lineage with respect to Cronobacter spp. epidemiology as $\mathrm{CC} 4$ has been identified as a genetic signature for the C. sakazakii meningitic pathovar. Previously Joseph and Forsythe announced the strong association between neonatal meningitis cases and C. sakazakii ST4 using a retrospective study of only 41 clinical strains from 1953 to 2008, collected from 7 countries [27]. This association was confirmed by the analysis of 15 USA cases in 2011 [26]. These two earlier predictions of the association of C. sakazakii CC4 with meningitis are confirmed in Table 2 where, from over a hundred clinical isolates, this clonal lineage is prevalent and is recovered more frequently than other sequence types from infant infections especially cases of meningitis (20/23 cases). The reason for the association of one clonal complex in the genus with neonatal meningitis is unclear at present as no particular virulence traits have

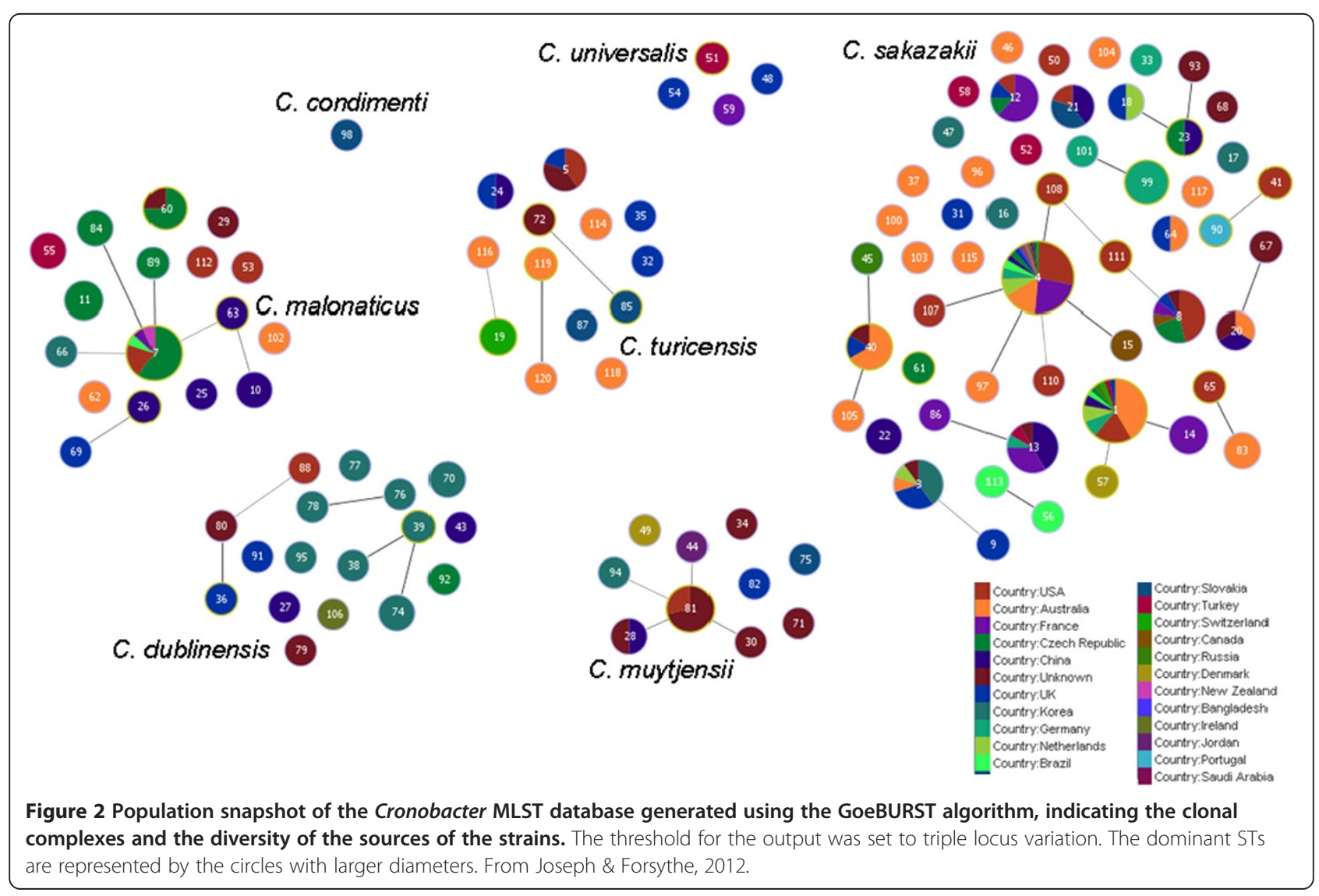


been determined in C. sakazakii CC4 compared to other sequence types [17].

It is notable that $24.6 \%(n=195)$ of C. sakazakii CC4 isolates were from infant formula and also that $16.9 \%$ of isolates were from environmental sources such as milk powder and infant formula manufacturing plants; Table 2. This supports the previous individual reports of isolates from in Ireland and Switzerland [28,29]. Sonbol et al. [30] reported that C. sakazakii CC4 accounted for $25 \%$ of Cronobacter strains isolated from the environment of 6 milk powder manufacturing plants in Australia and Germany, as well re-identified strains from an international survey in 1988 of Cronobacter in PIF. The finished genome sequence of a $C$. sakazakii CC4 strain (SP291) has been published, and was notable for its persistence in a PIF production facility in Ireland [29]. They also identified stress response and antimicrobial resistance genes in the genome. Furthermore, Muller et al. [28] used pulsed-field gel electrophoresis to match environmental isolates of C. sakazakii CC4 in a PIF manufacturing plant in Switzerland with those in the finished product. Therefore C. sakazakii CC4 may represent a particularly persistent clonal variant resulting in increased neonatal exposure. Whether the association of C. sakazakii CC4 with neonatal meningitis is due to greater neonatal exposure as a result of environmental fitness (i.e. desiccation persistence) or particular virulence capabilities is uncertain [17,27]. Therefore an improved understanding of $C$. sakazakii CC4 strains is warranted to understand its prevalence both in PIF manufacturing plants and severe neonatal infections. New genomic profiling of C. sakazakii CC4 strains is presented in the last section.

C. sakazakii clonal complex 1 (CC1) is the next prominent ST consisting of 80 strains (11\% of C. sakazakii strains in the database) isolated from around the world over a period of more than 25 years; Tables 1 and 2 . These have been isolated from PIF and from milk powder processing factories $[28,30]$. There are proportionately fewer $(12.5 \%) \mathrm{CC} 1$ clinical isolates compared to CC4 (45.1\%), and only three (3.8\%) of these strains are associated with meningitis [10]; Table 2. C. sakazakii CC1 strains were isolated from infant formula in 1994 used during a neonatal intensive care unit Cronobacter spp. outbreak in France [31]. Those strains did not match isolates from the infected neonates according to pulsed-field gel electrophoresis, and which were later shown to be C. sakazakii CC4 Also linked to this clonal complex is ST57 (DLV to ST1), which is the profile of a PIF isolate from Denmark in 1988. There are no known isolates of CC1 from adult patients which may indicate an adaptation to host by other clonal lineages.

Forty percent of $C$. malonaticus strains $(n=136)$ recorded in the database are in clonal complex 7. Strains in this complex have been isolated over the past 30 years. Within this complex $57 \%$ (33/58) of strains are clinical in origin and primarily from children and adults; Table 2. Only one reported fatal neonatal meningitis case has been attributed to $C$. malonaticus, though the vehicle of infection is uncertain and not necessarily linked to the consumption of infant formula [26]. The database only contains seven $C$. malonaticus CC7 isolates from PIF indicating a low incidence of this complex, and there are no isolates from infant formula or milk powder manufacturing plants.

Table 2 also shows for the first time that C. sakazakii ST12 has been associated with cases of necrotizing enterocolitis (13\% of strains) and not neonatal meningitis or septicaemia. Although there are isolates in the database from infant formula, unlike CC4 there have been none from milk powder or infant formula manufacturing plants. The remaining clonal groups in Table 2 are less clinically relevant, and are more food and environmental isolates.

The online Cronobacter MLST database has therefore enabled the open recording of sources of Cronobacter isolates and can be interrogated by researchers, industry and regulatory authorities. The recording of the isolates is standardized and therefore facilitates an international contribution to collating information.

\section{Ecology of Cronobacter spp.}

Table 2 shows all seven Cronobacter species have been isolated from food and food ingredients and these comprise $14.2 \%$ of the total profiled isolates. These are primarily from plant material including herbs and spices [22,32]. This is reflected in common physiological features which have been found in the genome [32-34]: yellow pigmentation for oxygen radical protection, capsule formation (adherence to plant surfaces) and efflux pumps (resistance to essential oils). Since plants are common food ingredients, these Cronobacter strains have been isolated from salads, cake mixes, packet soup, flavoured teas as well as powdered infant formula and weaning foods [35]. Table 2 shows that C. sakazakii CC4 strains are not so frequently isolated from food and food ingredients as $C$. malonaticus CC7; $8.2 \%$ compared to $25.9 \%$ of isolates. This may reflect the ecological diversity of the various clonal types and adaptation to niche. Another source for Cronobacter given in the database is water where 14/38 strains are $C$. dublinensis, and $13 / 38$ are C. sakazakii, with no $C$. sakazakii ST4 strains. Despite one reported serious infection which can be attributed to reconsititution with water containing Cronobacter, prior to this analysis, water as a source of the bacterium has not received much attention [26].

\section{Taxonomy of the Cronobacter genus}

Control of the organism requires a clear definition of the organism to distinguish it from closely related organisms 
which may be co-recovered. Accurate bacterial taxonomy is therefore essential for regulatory control because the detection methods must be based on a thorough understanding of the diversity of the target organism. A number of early Cronobacter detection methods were based on small numbers of poorly characterized, even misidentified, strains and therefore are not necessarily reliable for their stated purpose.

The application of NGS enabled an improved understanding of the taxonomy of the Cronobacter genus. The Cronobacter genus belongs to the bacterial class gammaproteobacteria, and is within the family Enterobacteriaceae with the nearest relatives being the newly described genus Kosakonia as well as the more familiar genera Citrobacter and Pantoea. Members of the latter include Citrobacter koseri which is notable as it is associated with invasive neonatal meningitis with clinical presentations of brain abscess formation. These symptoms are similar to those of Cronobacter spp. meningitis, and differ from those of the neonatal meningitis pathovar E. coli K1. Also members of the Pantoea spp. are primarily plant-borne and frequently are yellow pigmented as per the majority of Cronobacter isolates. Enterobacter hormaechei and E. ludwiggii isolates have been misidentified as Cronobacter which has led to some confusion in the literature [32].

Before the first (2004) FAO-WHO expert meeting yellow-pigmented Enterobacter cloacae-like bacterial strains were called Enterobacter sakazakii. Following further analysis, the Cronobacter genus was initially proposed for all previous E. sakazakii strains. The Cronobacter species were differentiated by Iversen et al. [15] according to $16 E$. sakazakii biotypes; C. sakazakii (biotypes $1-5,7,8,9,11$, 13 and 14), C. turicensis (biotypes 16, 16a and 16b), $C$. muytjensii (biotype 15), and C. dublinensis (biotypes 6, 10 and 12). This was quickly revised [36] with the addition of C. malonaticus (biotypes 5, 9 and 14). This latter species had originally been described as a sub-species of $C$. sakazakii by Iversen et al. [15] who could not distinguish C. sakazakii and C. malonaticus using $16 \mathrm{~S}$ rDNA sequence analysis. Distinguishing between the two species had been problematic primarily for two reasons. Firstly, the use of biotype profiles to designate the Cronobacter species was not totally robust as a few of the biotype index strains were themselves assigned the wrong species $[16,37]$. Secondly, there are seven copies of the rDNA gene in Cronobacter and intrageneric differences can lead to uncertain and inconsistent base calls with subsequent errors in GenBank entries.

In contrast, Joseph et al. [17] used strains selected by the seven loci multilocus sequence analysis (MLSA; 3036 bp concatenated sequence length) as representatives across the genus and therefore overcame the preconceived grouping of strains based on phenotyping. These studies led to the naming of two further Cronobacter species; $C$. universalis and C. condimenti [38] which as shown in Figure 1 are in the Cronobacter genus. Such recent changes in Cronobacter taxonomy are easily adapted using the sequence-based MLST scheme, whereas a number of currently approved methods including PCR probes require re-evaluation. In 2013, Brady et al. [39] used MLSA with only four loci $(g y r B, \operatorname{rpoB}$, infB and $a t p D$ ) for the reevaluation of the taxonomic status of Enterobacter helveticus, E. pulveris and E. turicensis. Consequently these were renamed C. helveticus, C. pulveris and C. zurichensis, respectively. This particular re-evaluation was debatable due to the limited number of loci used which is below the normal permitted number of five. This taxonomic change also cause problems with the international control of Cronobacter spp. in powdered infant formula as many detection methods were designed using these Enterobacter species as negative controls [40,41]. Soon after Stephan and colleagues proposed the latter three species should form two new genera named Franconibacter and Siccibacter [42]. Figure 1, using 7-loci MLSA confirms this taxonomic revision they do not cluster with the seven Cronobacter species.

The issue of whether all Cronobacter species are of clinical significance and need to be controlled in powdered infant formula, and other foods has not been considered by regulatory authorities. The lack of epidemiological evidence of infection from all species cannot be substantiated due to the frequent mis-identification of Cronobacter strains following the routine use of phenotyping for identification. However as shown in Table 2, only a few C. sakazakii and C. malonaticus clonal groups have been isolated from neonatal clinical cases. The other species are primarily environmental commensals and are probably of little clinical significance. Whether infections have been unreported due to misidentification is highly probable as commercially available phenotyping kits (approved in ISO and FDA detections method protocols) still use the name E. sakazakii, and these also identify many true Cronobacter isolates as Enterobacter cloacae, Pantoea spp. or Erwinia spp.

Many MLST databases are now implemented using the Bacterial Isolate Genome Sequence Database (BIGSdb) platform $[1,43]$. This enables a range of MLST-like schemes to be described and applied to whole genome sequenced organisms, in addition to the conventional laboratoryderived seven loci. Hence hierarchical classifications, either pre-defined in the database or user-defined, can be applied with progressively greater resolution as the number of loci analysed increases. In addition to the seven loci MLST scheme, as part of the Cronobacter site, a specific open-access repository for all Cronobacter genomes sequenced to date has been established. This enables the scalable analysis of Cronobacter genomes, representing all recognised species, for genes of interest 


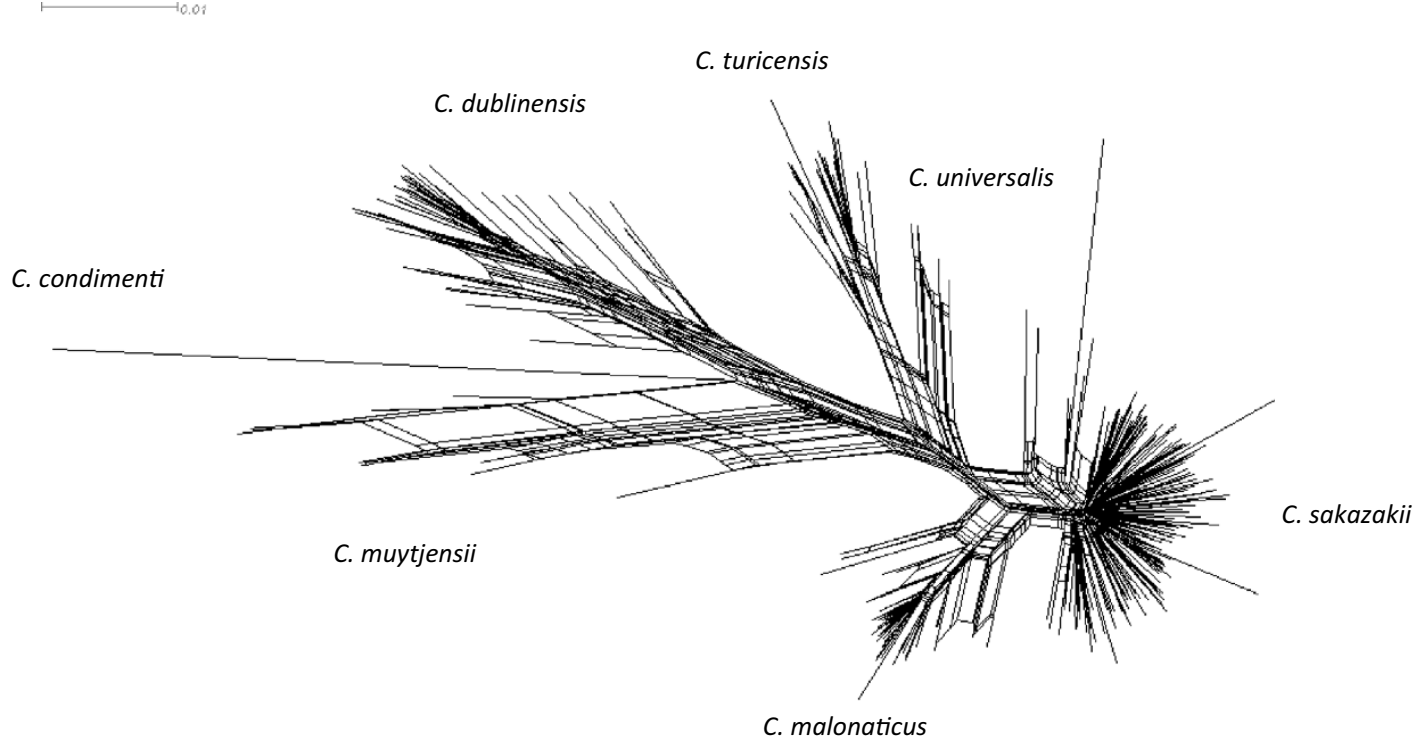

b. C. sakazakii and C. malonaticus with major clonal lineages highlighted

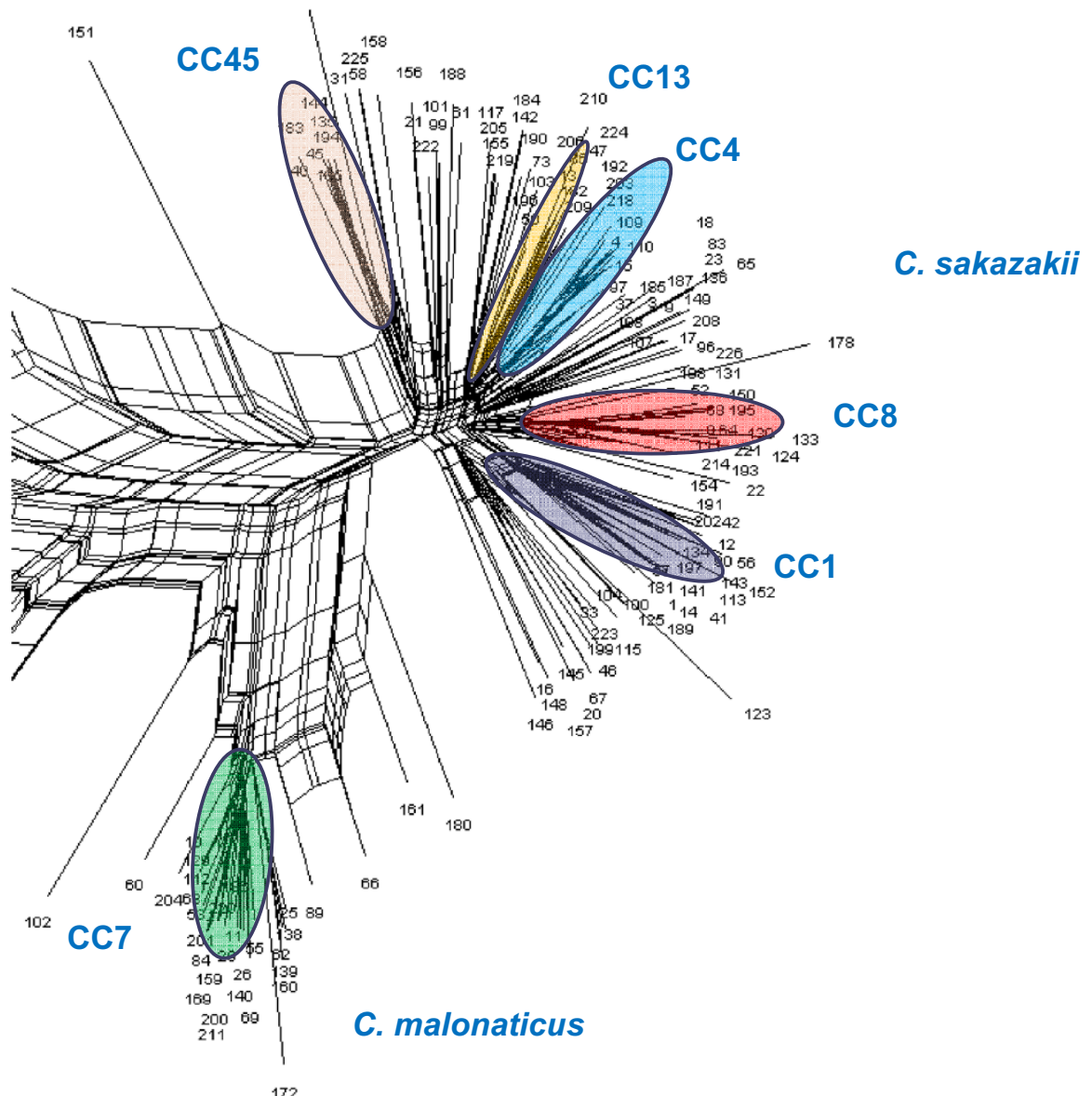

Figure 3 7-loci Splits Network of 298 sequence types from the Cronobacter genus. (a) Analyis of the whole Cronobacter genus (b) Analysis of C. sakazakii and C. malonaticus. 
and easily accommodates any changes in taxonomy. The Cronobacter seven loci MLST scheme has recently been extended online to include ompA and rроB sequences such that these loci can add to users' taxonomic evaluations (Tax-MLST).

The separation of species is not always easy to define, and a large number of recombination events can occur within species, resulting in multiple plausible but only partially consistent taxonomic trees. Consequently NeighborNet analysis using Splitstree [44] has been applied to the genome sequence data of Cronobacter. Whereas the separation of C. sakazakii and C. malonaticus using $16 \mathrm{~S}$ rRNA gene sequencing was problematic, the two species are clearly separated using the seven loci MLST (Figure $3 \mathrm{a}, \mathrm{b}$ ). The figure also shows the considerable genetic diversity of C. dublinensis and C. muytjensii which has not been reported before. This reflects that there is a relative large number of sequence types for these two species. As given in Table 1, although even combined they are only represent $9.4 \%$ of the recorded isolates, the 94 strains are composed of 58 sequence types. These species are primarily isolated from environmental sites and are of less clinical relevance. In contrast, C. sakazakii and C. malonaticus form distinct clusters, within which the C. sakazakii CC4 and C. malonaticus CC7 clonal complexes can be distinguished (Figure 3b).

Further multi-allelic analysis using genes coding for the ribosomal proteins (rMLST, 51-loci) has been used to integrate microbial genealogy and typing [2]. Expanding the multi-allelic analysis of Cronobacter spp. to rMLST shows the C. sakazakii CC4 clonal lineage as more pronounced and separate from the remaining $C$. sakazakii (Figure 4). This is based on 51 of the 53 ribosomal protein sequences due to the absence of two genes (rps $A$ and $r p s Q)$ in some strains in the sample group. This reveals the C. sakazakii ST4 clonal lineage as defined by just seven loci is robust, and is also found using 51 other housekeeping genes. The relevance being this sequence type is strongly associated with neonatal meningitis infections and has not been genotyped to this level before.

Furthermore, genes tagged as belonging to Clusters of Orthologous Genes (COG) can be used to generate COG-cgMLST with 1865 loci [45]. Further expansion of the genomic analysis using COG-defined genes of $C$. sakazakii ES15 as the reference genome generated 1865 loci which could be compared across the genus or selected species or strains. COG loci are identified by their being tagged as belonging to COGs in C. sakazakii ES15 (excluding loci named in other analysis schemes). The subsequent figure also highlights the $C$. sakazakii clonal lineage CC4; Figure 5. COG-cgMLST analysis can also be used to generate a profile of potential biochemical traits which may be of use for differentiating strains and species using phenotyping. This is particularly useful as previously the choice of biochemical tests to try has been arbitrary. In addition the scheme can be used to study a range of physiological traits. Table 3 summarises the presence and variation in genes associated with cell division [46]. The core FtsLBQ cell division complex and FtsE/FtsX $\mathrm{ABC}$ transporter components are found in all Cronobacter, Franconibacter and Siccibacter genomes, whereas ftsK is only present in C. sakazakii, $C$. malonaticus, $C$. universalis and $C$. turicensis. This variation in cell division proteins may be linked to environmental stress tolerance. Therefore the PubMLST scheme

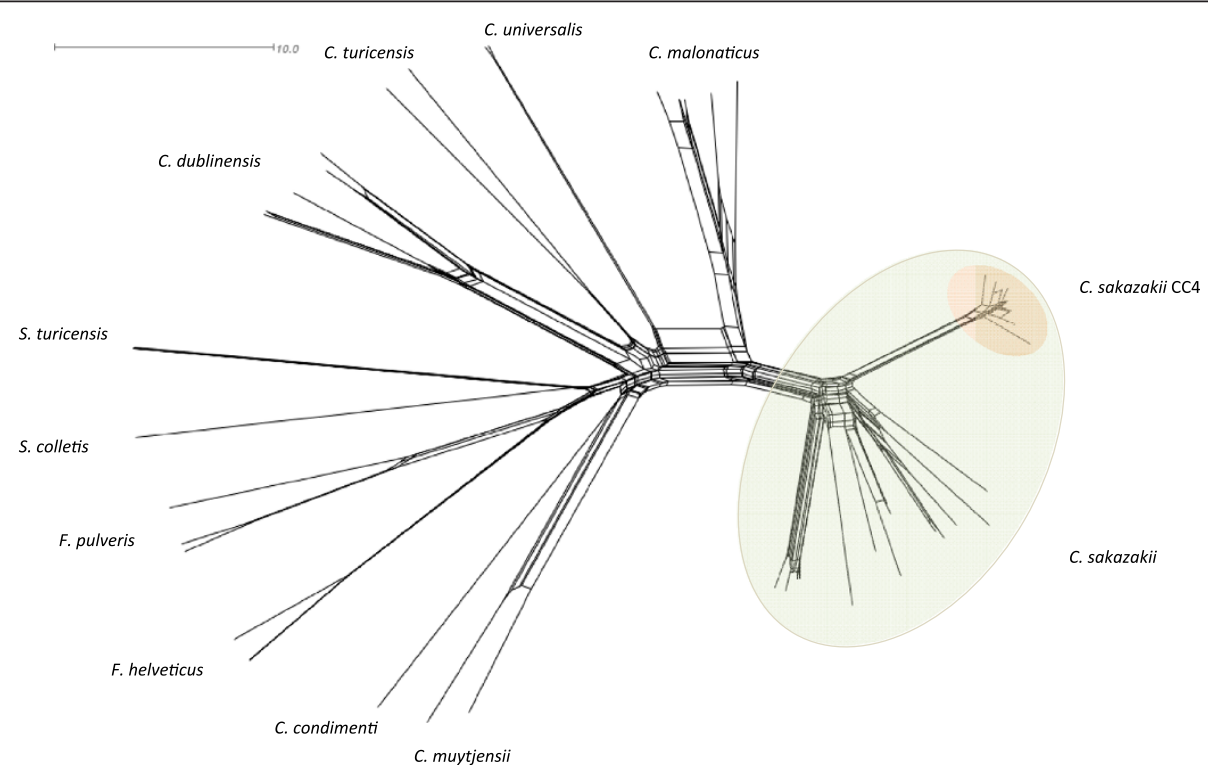

Figure 4 rMLST analysis of genomes across the Cronobacter, Franconibacter and Siccibacter genera. 


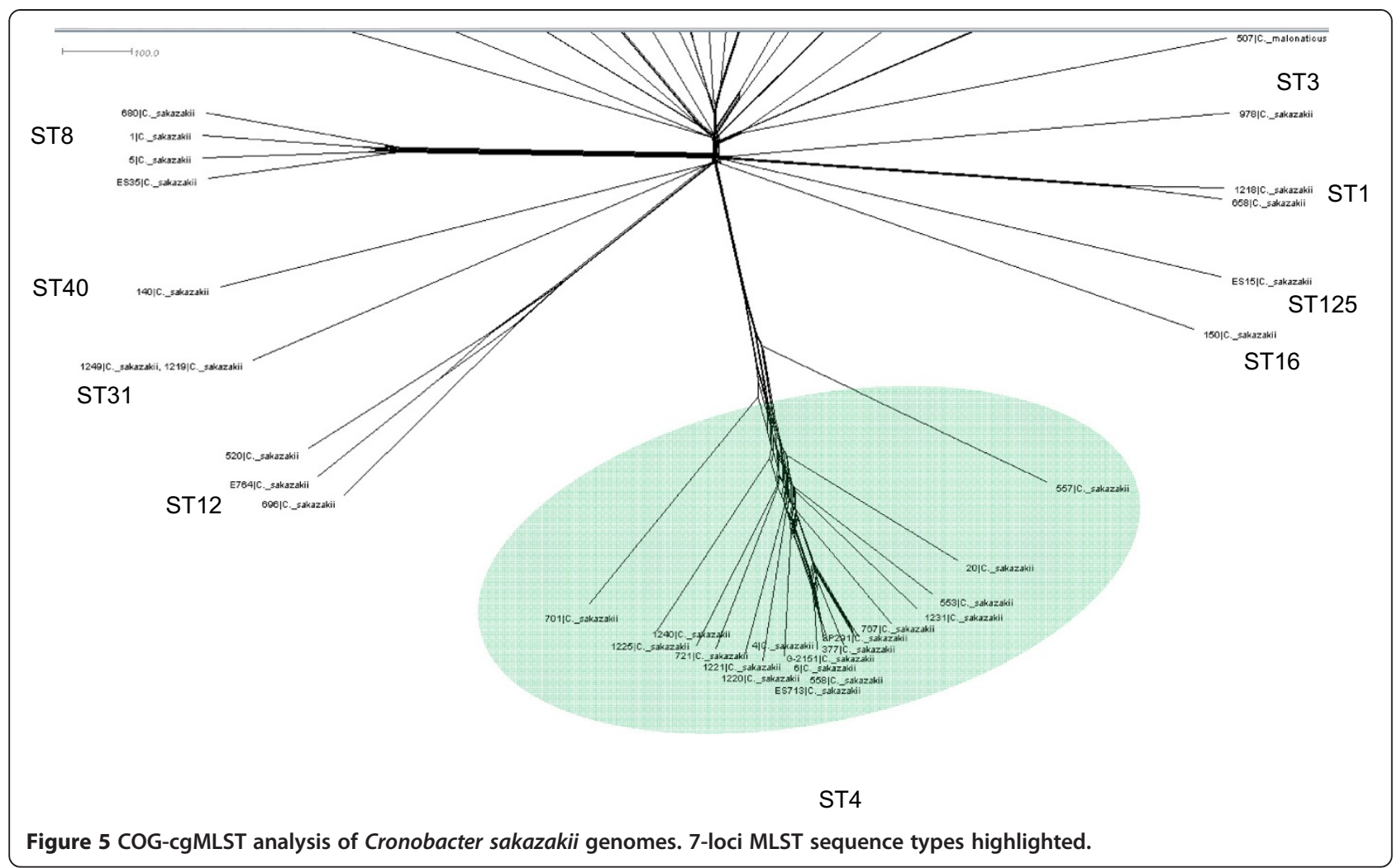

not only enables molecular typing of Cronobacter isolates, but also readily accessible investigative profiling of strains.

\section{Cronobacter spp. virulence traits}

Cronobacter can invade human intestinal cells, replicate in macrophages, and invade the blood brain barrier $[47,48]$. The route of infection is probably through attachment and invasion of the intestinal cells. Whole genome sequencing has revealed a large number of plausible virulence factors, though many require further laboratory studies for confirmation [32-34]. Additionally the genomes of 107 Cronobacter strains, including 50 C. sakazakii ST4, are available for BLAST analysis and comparative genomic analysis via the PubMLST database.

Ten fimbriae clusters have been identified in the genomes of Cronobacter species [34]. Many fimbriae clusters are common to all species, though there are two interesting exceptions. C. sakazakii is the only Cronobacter species encoding for $\beta$-fimbriae, whereas the genomes of the other species encode for curli fimbriae. This may reflect evolution to the host ecosystem. A number of iron assimilation mechanisms have been found in Cronobacter species [49] which might enable the organism to utilize iron from breast milk and formula. Five putative Type VI secretion system (T6SS) clusters have been identified in Cronobacter spp. genomes. These may be involved in adherence, cytotoxicity, host-cell invasion, growth inside macrophages and survival within the host. It has been proposed that the outer membrane proteins ompA and ompX have roles in Cronobacter penetrating the blood brain barrier [50]. The mechanism(s) leading to the destruction of the brain cells is unknown and could in part be a host response. The organism also encodes for a number of haemolysins [34]. The organism has several plasmid borne features of interest including encoding for an outer membrane protease (Cronobacter plasminogen activator) that has significant identity to proteins belong to the Pla subfamily of omptins. Members of this subfamily of proteins degrade a number of serum proteins, including circulating complement, providing protection from the complementdependent serum killing [51]. Having 107 genomes representing the Cronobacter genus in a curated, openaccess database will facilitate the further analysis of these and other virulence traits. For example, using the BLAST facility shows the cpa gene is present in C. sakazakii strains ( $\mathrm{n}=72$ genomes) but not $C$. malonaticus ( $\mathrm{n}=14$ genomes) which may partially explain the variation in host susceptibility.

\section{Other Cronobacter characteristics of interest}

Comparative genomic studies revealed that C. sakazakii possessed the nanATKR gene cluster which encodes for the utilization of exogenous sialic acid, and this may have clinical significance $[34,52]$. The ability to utilise sialic acid as a carbon source could be a major evolutionary hostadaptation since the compound is found in breast milk, 
Table 3 Presence of cell division genes across the Cronobacter genus and closely related genera Franconibacter and Siccibacter

\begin{tabular}{|c|c|c|c|c|c|c|c|c|c|c|c|c|}
\hline Gene & Function & $\begin{array}{l}\text { C. sakazakii } \\
(33)^{\mathrm{a}}\end{array}$ & $\begin{array}{l}\text { C. malonaticus } \\
\text { (5) }\end{array}$ & $\begin{array}{l}\text { C. universalis } \\
\text { (2) }\end{array}$ & $\begin{array}{l}\text { C. turicensis } \\
\text { (2) }\end{array}$ & $\begin{array}{l}\text { C. muytjensii } \\
\text { (2) }\end{array}$ & $\begin{array}{l}\text { C. dublinensis } \\
\text { (5) }\end{array}$ & $\begin{array}{l}\text { C. condimenti } \\
\text { (1) }\end{array}$ & $\begin{array}{l}\text { F. helveticus } \\
\text { (2) }\end{array}$ & $\begin{array}{l}\text { F. pulveris } \\
\text { (3) }\end{array}$ & $\begin{array}{l}\text { S. turicensis } \\
\text { (3) }\end{array}$ & $\begin{array}{l}\text { Candidatus S. } \\
\text { colletis (1) }\end{array}$ \\
\hline$f t s L$ & $\begin{array}{l}\text { FtsLBQ cell division } \\
\text { complex }\end{array}$ & 33 & 5 & 2 & 2 & 2 & 5 & 1 & 2 & 3 & 3 & 1 \\
\hline $\mathrm{fts} B$ & $\begin{array}{l}\text { FtsLBQ cell division } \\
\text { complex }\end{array}$ & 33 & 5 & 2 & 2 & 2 & 5 & 1 & 2 & 3 & 3 & 1 \\
\hline $\mathrm{fts} Q$ & $\begin{array}{l}\text { FtsLBQ cell division } \\
\text { complex }\end{array}$ & 33 & 5 & 2 & 2 & 2 & 5 & 1 & 2 & 3 & 3 & 1 \\
\hline $\mathrm{fts} Y$ & $\begin{array}{l}\text { Signal recognition particle } \\
\text { protein translocation } \\
\text { system }\end{array}$ & 32 & 5 & 2 & 2 & 2 & 5 & 0 & 2 & 3 & 3 & 1 \\
\hline ftsE & FtsE/FtsX ABC transporter & 33 & 5 & 2 & 2 & 2 & 5 & 1 & 2 & 3 & 3 & 1 \\
\hline$f t s \times 1$ & FtsE/FtsX ABC transporter & 33 & 5 & 2 & 2 & 2 & 5 & 1 & 2 & 3 & 3 & 1 \\
\hline$f t s \times 2$ & FtsE/FtsX ABC transporter & 28 & 5 & 2 & 2 & 1 & 4 & 1 & 2 & 3 & 3 & 1 \\
\hline$f t s l$ & $\begin{array}{l}\text { Penicillin-binding } \\
\text { protein } 3\end{array}$ & 33 & 5 & 2 & 2 & 2 & 5 & 1 & 2 & 3 & 3 & 1 \\
\hline $\mathrm{ftsW}$ & Lipid II flippase & 33 & 5 & 2 & 2 & 2 & 5 & 1 & 2 & 3 & 3 & 1 \\
\hline $\mathrm{fts} A$ & $\begin{array}{l}\text { Essential cell division } \\
\text { protein }\end{array}$ & 33 & 5 & 2 & 2 & 2 & 5 & 1 & 2 & 3 & 3 & 1 \\
\hline $\mathrm{fts} Z$ & $\begin{array}{l}\text { Essential cell division } \\
\text { protein }\end{array}$ & 33 & 5 & 2 & 2 & 2 & 5 & 1 & 2 & 3 & 3 & 1 \\
\hline zipA & $\begin{array}{l}\text { Essential cell division } \\
\text { protein }\end{array}$ & 33 & 5 & 2 & 2 & 2 & 5 & 1 & 0 & 0 & 0 & 0 \\
\hline$f t s N$ & Uncertain & 33 & 5 & 2 & 2 & 2 & 5 & 1 & 0 & 3 & 0 & 0 \\
\hline $\mathrm{ftsK}$ & DNA translocase & 29 & 5 & 1 & 2 & 0 & 0 & 0 & 0 & 0 & 0 & 0 \\
\hline
\end{tabular}

${ }^{a}$ Number of genomes analysed. 
mucin and gangliosides. Sialic acid is also an ingredient in powdered infant formula due to its association with brain development. Laboratory studies designed to confirm the sialic acid utilization was active in C. sakazakii and also showed the species was able to grow on the ganglioside GM1 as a sole carbon source [52]. In contrast, unlike other Cronobacter species, C. sakazakii is unable to use malonic acid. Genomic analysis shows the malonate decarboxylase genes are next to an auxin efflux carrier which may function in malonate uptake. Malonic acid is an organic acid which is found in plant tissues and reflects the plant-association of the Cronobacter genus. The adaptation of C. sakazakii to a new ecosystem, with the subsequent loss of malonate utilization and gain in sialic acid utilization might contribute to this clinical significance. Cronobacter genomes also show the presence of a number of heavy metal resistance traits (copper, silver, zinc, tellurite) and capsule formation which might enable it to resist disinfectants in food production environments.

Hence the Cronobacter PubMLST database enabled researchers to study the detailed ecology, taxonomy and virulence of the organism. A timeline of key Cronobacter events are given in Additional file 1: Box 3, and list the various contributions of the Cronobacter PubMLST database.

\section{Conclusions}

This paper demonstrates the application of NGS to Cronobacter, an emergent bacterial pathogen which had been poorly defined as a single species Enterobacter sakazakii. The advantages of a centralised multilocus sequence typing (MLST) database (i.e., http://pubmlst.org/ cronobacter/) for genotyping the organism and recognising clinically relevant clonal lineages has been demonstrated using just seven loci. These lineages have been confirmed by 51-loci rMLST and even whole genome allelic profiling to the clone level. In addition, the three FAO-WHO key requests have been addressed by implementing this database using the BIGSdb platform, enabling the inclusion of 107 complete genomes within the 1007 recorded isolates. This database has also enabled the retrospective analysis of historic cases and outbreaks following re-identification of those strains and hence minimized the loss in information following taxonomic re-evaluations.

The multilocus sequence typing (MLST) and analysis (MLSA) approaches have:

a. Revealed the diversity of the genus

b. Contributed to the recognition of new species

c. Shown the relatedness of the species,

d. Shown the evolutionary decent of the genus

e. Revealed the majority of neonatal meningitis cases are being attributable to one clonal lineage, $C$. sakazakii ST4 (CC4) f. Form the basis for future research regarding strain selection for investigating Cronobacter virulence and environmental fitness.

The Cronobacter PubMLST database offers a central, open access, reliable sequence-based repository. It has the capacity to create new analysis schemes 'on the fly' and integrate metadata (source, geographic distribution, clinical presentation). The database is able to adapt to changes in taxonomy and offers expandable analysis through BIGSdb implementation.

By producing a phylogenetic network for increasingly large fractions of the genome, rMLST and COGcgMLST facilitate the identification of sequence types and species, while illustrating the ambiguities inherent in phylogenetic reconstruction across a genus. The application of Next Generation Sequencing has not only met the objectives of the 2004 FAO-WHO tasks, but continues to exceed them as the number of sequenced genomes has progressed.

\section{Methods}

\section{Source DNA sequences}

DNA sequences collated at http://pubmlst.org/cronobacter/ were investigated and Table 1 gives a summary of these strains. In silico analyses were carried out using options on the Cronobacter PubMLST portal accessible at: http://pubmlst.org/perl/bigsdb/bigsdb.pl?db=pubmlst_ cronobacter_isolates.

\section{Seven loci MLST analysis}

Concatenated sequences of seven loci from 298 sequence types were downloaded in FASTA format using the Export/Sequences option. These sequences were aligned in MEGA version 6.05 using the ClustalW algorithm. The final alignment spanned $3036 \mathrm{bp}$ and was analysed using the default pipeline in SplitsTree4 (UncorrectedP to calculate distances and NeighborNet to build the network).

\section{rMLST analysis}

A distance matrix for 107 Cronobacter genomes with available whole-genome sequences (and representing the whole genus [2]) was calculated with the Analysis/Genome Comparator option using default settings (with tagged allele designations used if available and completely excluding truncated loci). The matrix was exported in nexus format and analysed using the default pipeline in SplitsTree 4 (as above).

\section{COG-cgMLST scheme set-up and analysis}

COG-cgMLST uses genes annotated as belonging to Clusters of Orthologous Groups [45] to define 1865 loci. The locus list, including reference sequences from Cronobacter sakazakii ES15, was assembled using an in-house 
script which identified CDSs in Genbank accession CP003312.1 in which the $<$ note $>$ field included a COG identifier. Sequences from these tagged loci were extracted and uploaded to the Cronobacter sequence definitions database excluding loci already named therein. Further scripts were used to establish COG-cgMLST locus scheme initially containing 1866 loci. The scheme was subsequently edited to exclude the locus ppsA (identical to $p p s$ in MLST) yielding 1865 loci.

With the scheme established, analysis was carried out as above with the use of the Analysis/Genome Comparator option and the default pipeline in SplitsTree4 (as above). Note that the Genome Comparator option with COG-cgMLST will analyse samples directly against the reference-defined loci and not with reference to COG IDs in query genomes.

\section{Additional file}

Additional file 1: Box 1. FAO-WHO executive summary (2004); see footnote. Box 2. Box 3. Summary timeline of Cronobacter recognition, control and molecular profiling [53].

\begin{abstract}
Abbreviations
BIGSdb: Bacterial Isolate Genome Sequence Database; CC: Clonal complex; cgMLST: Core genome multilocus sequence typing; COG: Clusters of Orthologous Groups; COG-cgMLST: Clusters of Orthologous Groups-core genome multilocus sequence typing; FAO-WHO: Food and Agricultural Organization-World Health Organization; MLSA: Multilocus sequence analysis; MLST: Multilocus sequence typing; NGS: Next Generation Sequencing; NEC: Necrotizing enterocolitis; PIF: Powdered infant formula; rMLST: Ribosomal multilocus sequence typing; ST: Sequence types; Tax-MLST: Taxonomic multilocus sequence typing; wgMLST: Whole genome multilocus sequence typing.
\end{abstract}

\section{Competing interests}

The authors declare that they have no competing interests.

\section{Authors' contributions}

SF conceived of the study, and participated in its design, coordination and drafted the manuscript. BD participated in the sequence analysis and helped to draft the manuscript. KJ participated in the sequence analysis and helped to draft the manuscript. All authors read and approved the final manuscript.

\section{Acknowledgements}

This publication made use of the Cronobacter Multi Locus Sequence Typing website (http://pubmlst.org/cronobacter/) developed by Keith Jolley and sited at the University of Oxford [46]. The development of this site has been funded by the Wellcome Trust. The authors thank our numerous collaborators especially those involved who have submitted sequences to the Cronobacter MLST database, and Pauline Ogrodzki, Emily Jackson, Sumyya Hariri and Abdlrhman Alsonosi for their assistance in preparing this manuscript.

\section{Author details}

'School of Science and Technology, Nottingham Trent University, Clifton Lane, Nottingham NG11 8NS, UK. ²Department of Zoology, University of Oxford, Oxford OX1 3PS, UK

Received: 3 July 2014 Accepted: 11 December 2014

Published: 16 December 2014

\section{References}

1. Maiden MC, van Rensburg MJ, Bray JE, Earle SG, Ford SA, Jolley KA, McCarthy ND: MLST revisited: The gene-by-gene approach to bacterial genomics. Nat Rev Microbiol 2013, 11:728-736.

2. Jolley KA, Bliss CM, Bennett JS, Bratcher HB, Brehony C, Colles FH, Wimalarathna H, Harrison OB, Sheppard SK, Cody AJ, Maiden MCJ: Ribosomal multilocus sequence typing: universal characterization of bacteria from domain to strain. Microbio/ 2012, 158:1005-1015.

3. Jolley KA, Maiden MC: Automated extraction of typing information for bacterial pathogens from whole genome sequence data: Neisseria meningitidis as an exemplar. Euro Surveill 2013, 18:pii=20379.

4. Sheppard SK, Jolley KA, Maiden MJC: A gene-by-gene approach to bacterial population genomics: whole genome MLST of Campylobacter. Genes 2012, 3:261-277.

5. Pérez-Losada M, Cabezas P, Castro-Nallar E, Crandall KA: Pathogen typing in the genomics era: MLST and the future of molecular epidemiology. Infect Gen Evol 2013, 16:38-53.

6. Leopold SR, Goering RV, Witten A, Harmsen D, Mellmann A: Bacterial whole-genome sequencing revisited: portable, scalable, and standardized analysis for typing and detection of virulence and antibiotic resistance genes. J Clin Microbiol 2014, 52:2365-2370.

7. van Acker J, de Smet F, Muyldermans G, Bougatef A, Naessens A, Lauwers S: Outbreak of necrotizing enterocolitis associated with Enterobacter sakazakii in powdered milk formula. J Clin Microbiol 2001, 39:293-297.

8. Himelright I, Harris E, Lorch V, Anderson M: Enterobacter sakazakii infections associated with the use of powdered infant formula-Tennessee, 2001. J Am Med Assoc 2002, 287:2204-2205.

9. Iversen C, Forsythe S: Risk profile of Enterobacter sakazakii, an emergent pathogen associated with infant milk formula. T Food Sci Technol 2003, 14:443-454.

10. Food and Agricultural Organization-World Health Organization: Enterobacter Sakazakii And Other Organisms In Powdered Infant Formula. In Microbiological Risk Assessment Series 6. Geneva; 2004 [http://www. who.int/foodsafety/publications/mra6-enterobacter-sakazakii/en/]

11. Food and Agricultural Organization-World Health Organization: Enterobacter Sakazakii And Salmonella In Powdered Infant Formula. In Microbiological Risk Assessment Series 10. Rome; 2006 [http://www.who.int/foodsafety/publications/mra10/en/]

12. Food and Agricultural Organization-World Health Organization: Enterobacter Sakazakii (Cronobacter spp.) In Powdered Follow-Up Formula. In Microbiological Risk Assessment Series 15. Washington; 2008 [http://www. who.int/foodsafety/publications/mra_followup/en/]

13. Farmer JJ, Asbury MA, Hickman FW, Brenner DJ: Enterobacter sakazakii: a new species of "Enterobacteriaceae" isolated from clinical specimens. Intl J Syst Bacteriol 1980, 30:569-584.

14. Iversen C, Waddington M, On SLW, Forsythe S: Identification and phylogeny of Enterobacter sakazakii relative to Enterobacter and Citrobacter. J Clin Microbiol 2004, 42:5368-5370.

15. Iversen C, Lehner A, Mullane N, Bidlas E, Cleenwerck I, Marugg J, Joosten H: The taxonomy of Enterobacter sakazakii: Proposal of a new genus Cronobacter gen. nov. and descriptions of Cronobacter sakazakii comb. nov. Cronobacter sakazakii subsp. sakazakii, comb. nov., Cronobacter sakazakii subsp. malonaticus subsp. nov., Cronobacter turicensis sp. nov., Cronobacter muytjensii sp. nov., Cronobacter dublinensis sp. nov. and Cronobacter genomospecies 1. BMC Evol Biol 2007, 7:64.

16. Baldwin A, Loughlin M, Caubilla-Barron J, Kucerova E, Manning G, Dowson C, Forsythe S: Multilocus sequence typing of Cronobacter sakazakii and Cronobacter malonaticus reveals stable clonal structures with clinical significance which do not correlate with biotypes. BMC Microbiol 2009, 9:223.

17. Joseph S, Sonbol H, Hariri S, Desai P, McClelland M, Forsythe SJ: Diversity of the Cronobacter genus as revealed by multilocus sequence typing. J Clin Microbiol 2012, 50:3031-3039.

18. Codex Alimentarius Commission: Code of hygienic practice for powdered formulae for infants and young children. CAC/RCP 2008, 66:1-29.

19. Cetinkaya E, Joseph S, Ayhan K, Forsythe SJ: Comparison of methods for the microbiological identification and profiling of Cronobacter species from ingredients used in the preparation of infant formula. Mol Cell Probes 2013, 27:60-64.

20. Iversen C, Druggan P, Forsythe SJ: A selective differential medium for Enterobacter sakazakii. Intl J Food Microbiol 2004, 96:133-139. 
21. Masood N, Moore K, Farbos A, Hariri S, Paszkiewicz K, Dickins B, McNally A, Forsythe S: Draft genome sequence of the earliest Cronobacter sakazakii sequence type 4 strain NCIMB 8272. Genome Announc 2013, 2:e00585-14.

22. Iversen C, Forsythe SJ: Isolation of Enterobacter sakazakii and other Enterobacteriaceae from powdered infant formula milk and related products. Food Microbiol 2004, 21:771-776.

23. Holy O, Forsythe SJ: Cronobacter species as emerging causes of healthcare-associated infection. J Hosptl Infect 2014, 86:169-177.

24. Hurrell E, Kucerova E, Loughlin M, Caubilla-Barron J, Hilton A, Armstrong R, Smith C, Grant J, Shoo S, Forsythe S: Neonatal enteral feeding tubes as loci for colonisation by members of the Enterobacteriaceae. BMC Infect Dis 2009, 9:146.

25. Joseph S, Forsythe SJ: Insights into the emergent bacterial pathogen Cronobacter spp., generated by multilocus sequence typing and analysis. Front Food Microbiol 2012, 3:397.

26. Hariri S, Joseph S, Forsythe SJ: Cronobacter sakazakii ST4 strains and neonatal meningitis, US. Emerg Inf Dis 2013, 19:175-177.

27. Joseph S, Forsythe S: Predominance of Cronobacter sakazakii sequence type 4 in neonatal infections. Emerg Inf Dis 2011, 17:1713-1715.

28. Muller A, Stephan R, Fricker-Feer C, Lehner A: Genetic diversity of Cronobacter sakazakii isolates collected from a Swiss infant formula production facility. J Food Protect 2013, 76:883-887.

29. Power KA, Yan Q, Fox EM, Cooney S, Fanning S: Genome sequence of Cronobacter sakazakii SP291, a persistent thermotolerant isolate derived from a factory producing powdered infant formula. Genome Announc 2013, 1:13.

30. Sonbol H, Joseph S, McAuley C, Craven H, Forsythe SJ: Multilocus sequence typing of Cronobacter spp. from powdered infant formula and milk powder production factories. Intl Dairy J 2013, 30:1-7.

31. Caubilla-Barron J, Hurrell E, Townsend S, Cheetham P, Loc-Carrillo C, Fayet O, Prere M-F, Forsythe SJ: Genotypic and phenotypic analysis of Enterobacter sakazakii strains from an outbreak resulting in fatalities in a neonatal intensive care unit in France. J Clin Microbiol 2007, 45:3979-3985.

32. Kucerova E, Joseph S, Forsythe S: Cronobacter: diversity and ubiquity. Qual Ass Safety Foods Crops 2011, 3:104-122.

33. Kucerova E, Clifton SW, Xia X-Q, Long F, Porwollik S, Fulton L, Fronick C, Minx P, Kyung K, Warren W, Fulton R, Feng D, Wollam A, Shah N, Bhonagiri V, Nash WE, Hallsworth-Pepin K, Wilson RK, McClelland M, Forsythe SJ: Genome sequence of Cronobacter sakazakii BAA-894 and comparative genomic hybridization analysis with other Cronobacter species. PLoS One 2010, 5:e9556.

34. Joseph S, Desai P, Ji Y, Cummings CA, Shih R, Degoricija L, Forsythe SJ: Comparative analysis of genome sequences covering the seven Cronobacter species. PLoS One 2012, 7:e49455.

35. Chap J, Jackson P, Siqueira R, Gaspar N, Quintas C, Park J, Osaili T, Shaker R, Jaradat Z, Hartantyo SHP, Abdullah Sani N, Estuningsih S, Forsythe SJ: International survey of Cronobacter sakazakii and other Cronobacter spp. in follow up formulas and infant foods. Int/ J Food Microbiol 2009, 136:185-188.

36. Iversen C, Mullane N, McCardell B, Tall BD, Lehner A, Fanning S, Joosten H: Cronobacter gen. nov., a new genus to accommodate the biogroups of Enterobacter sakazakii, and proposal of Cronobacter sakazakii gen. nov., comb. nov., Cronobacter malonaticus sp. nov., Cronobacter turicensis sp. nov., Cronobacter muytjensii sp. nov., Cronobacter dublinensis sp. nov., Cronobacter genomospecies 1, and of three subspecies, Cronobacter dublinensis subsp. dublinensis subsp. nov., Cronobacter dublinensis subsp. lausannensis subsp. nov. and Cronobacter dublinensis subsp. lactaridi subsp. nov. Intl J Syst Evol Microbiol 2008, 58:1442-1447.

37. Joseph S, Hariri S, Forsythe SJ: Lack of continuity between Cronobacter biotypes and species as determined using multilocus sequence typing. Mol Cell Probes 2013, 27:137-139.

38. Joseph S, Cetinkaya E, Drahovska H, Levican A, Figueras MJ, Forsythe SJ: Cronobacter condimenti sp. nov., isolated from spiced meat, and Cronobacter universalis sp. nov., a species designation for Cronobacter sp. genomospecies 1, recovered from a leg infection, water and food ingredients. Int/ J Syst Evol Microbiol 2012, 62:1277-1283.

39. Brady C, Cleenwerck I, Venter S, Coutinho T, De Vos P: Taxonomic evaluation of the genus Enterobacter based on multilocus sequence analysis (MLSA): Proposal to reclassify E. nimipressuralis and E. amnigenus into Lelliottia gen. nov. as Lelliottia nimipressuralis comb. nov. and Lelliottia amnigena comb. nov., respectively, E. gergoviae and E. pyrinus into Pluralibacter gen. nov. as Pluralibacter gergoviae comb. nov. and Pluralibacter pyrinus comb. nov., respectively, E. cowanii, E. radicincitans, E. oryzae and E. arachidis into Kosakonia gen. nov. as Kosakonia cowani comb. nov., Kosakonia radicincitans comb. nov., Kosakonia oryzae comb. nov. and Kosakonia arachidis comb. nov., respectively, and E. turicensis, E. helveticus and E. pulveris into Cronobacter as Cronobacter zurichensis nom. nov., Cronobacter helveticus comb. nov. and Cronobacter pulveris comb. nov., respectively, and emended description of the genera Enterobacter and Cronobacter. Syst Appl Microbiol 2013, 36:309-319.

40. Masood N, Jackson E, Moore K, Farbos A, Paszkiewicz K, Dickins B, McNally A, Forsythe S: Draft genome sequence of "Candidatus Cronobacter colletis" NCTC $14934^{\top}$, a new species in the genus Cronobacter. Genome Announc 2014, 2:e00585-14.

41. Jackson EE, Sonbol H, Masood N, Forsythe SJ: Genotypic and phenotypic characteristics of Cronobacter species, with particular attention to the newly reclassified species $C$. helveticus, $C$. pulveris, and $C$. zurichensis. Food Microbiol 2014, 44:226-235.

42. Stephan R, Grim CJ, Gopinath GR, Mammel MK, Sathyamoorthy V, Trach LH, Chase HR, Fanning S, Tall BD: Re-examination of the taxonomic status of Enterobacter helveticus, Enterobacter pulveris, and Enterobacter turicensis as members of Cronobacter and description of Siccibacter turicensis com. nov., Franconibacter helveticus comb. nov., and Franconibacter pulveris com. nov. Int J Syst Evol Microbiol 2014, 64:3402-3410.

43. Jolley KA, Maiden MC: BIGSdb: Scalable analysis of bacterial genome variation at the population level. BMC Bioinform 2010, 11:595.

44. Huson DH, Bryant D: Application of phylogenetic networks in evolutionary studies. Mol Biol Evol 2006, 23:254-267.

45. Tatusov RL, Galperin MY, Natale DA, Koonin EV: The COG database: a tool for genome-scale analysis of protein functions and evolution. Nucleic Acids Res 2000, 28:33-36.

46. Samaluru H, SaiSree L, Reddy M: Role of Sufl (FtsP) in cell division of Escherichia coli: evidence for its involvement in stabilizing the assembly of the divisome. J Bacteriol 2007, 189:8044-8052.

47. Townsend SM, Hurrell E, Gonzalez-Gomez I, Lowe J, Frye JG, Forsythe S, Badger JL: Enterobacter sakazakii invades brain capillary endothelial cells, persists in human macrophages influencing cytokine secretion and induces severe brain pathology in the neonatal rat. Microbiology 2007 153:3538-3547.

48. Townsend SM, Hurrell E, Forsythe S: Virulence studies of Enterobacter sakazakii isolates associated with a neonatal intensive care unit outbreak. BMC Microbiol 2008, 8:64

49. Grim CJ, Kothary MH, Gopinath G, Jarvis KG, Beaubrun JJ, McClelland M, Tall BD, Franco AA: Identification and characterization of Cronobacter iron acquisition systems. Appl Environ Microbiol 2012, 78:6035-6050.

50. Kim K, Kim KP, Choi J, Lim JA, Lee J, Hwang S, Ryu S: Outer membrane proteins $\mathrm{A}(\mathrm{OmpA})$ and $\mathrm{X}(\mathrm{OmpX})$ are essential for basolateral invasion of Cronobacter sakazakii. Appl Env Microbiol 2010, 76:5188-5198.

51. Franco AA, Kothary MH, Gopinath G, Jarvis KG, Grim CJ, Hu L, Datta AR, McCardell BA, Tall BD: Cpa, the outer membrane protease of Cronobacter sakazakii, activates plasminogen and mediates resistance to serum bactericidal activity. Infect Immun 2011, 79:1578-1587.

52. Joseph S, Hariri S, Masood N, Forsythe S: Sialic acid utilization by Cronobacter sakazakii. Microbial Inform Exptn 2013, 3:3.

53. Chen Y, Lampel K, Hammack T: Bacteriological Analytical Manual, Chapter 29. In Cronobacter; [http://www.fda.gov/Food/FoodScienceResearch/ LaboratoryMethods/ucm289378.htm]

\section{doi:10.1186/1471-2164-15-1121}

Cite this article as: Forsythe et al: Cronobacter, the emergent bacterial pathogen Enterobacter sakazakii comes of age; MLST and whole genome sequence analysis. BMC Genomics 2014 15:1121. 